\title{
A phenomics approach to the analysis of the influence of glutathione on leaf area and abiotic stress tolerance in Arabidopsis thaliana
}

\author{
Daniel Schnaubelt ${ }^{1}$, Philipp Schulz ${ }^{2+}$, Matthew A. Hannah ${ }^{2}$, Rosita E. Yocgo ${ }^{1,3+}$ and Christine H. Foyer ${ }^{1}$ * \\ ${ }^{1}$ Centre of Plant Sciences, Faculty of Biology, University of Leeds, Leeds, UK \\ ${ }^{2}$ Bayer CropScience NV, Gent, Belgium \\ ${ }^{3}$ Botany Department, Forestry and Agricultural Biotechnology Institute, University of Pretoria, Pretoria, South Africa
}

\author{
Edited by: \\ Jean-Philippe Reichheld, Centre \\ national de la recherche scientifique, \\ France \\ Reviewed by: \\ Xinguang Zhu, Chinese Academy \\ of Sciences, China \\ Vasileios Fotopoulos, Cyprus \\ University of Technology, Cyprus \\ ${ }^{*}$ Correspondence: \\ Christine H. Foyer, Centre of Plant \\ Sciences, School of Biology, Faculty \\ of Biological Sciences, University of \\ Leeds, Leeds LS2 9JT, UK \\ e-mail: c.foyer@leeds.ac.uk \\ ${ }^{\dagger}$ Present address: \\ Rosita E. Yocgo, Institute for Plant \\ Biotechnology, University of \\ Stellenbosch, Private Bag X1, \\ Matieland 7602, South Africa; \\ Philipp Schulz, Department of Plant \\ Biochemistry, Dahlem Centre of Plant \\ Sciences, Freie Universität Berlin, \\ 14195 Berlin, Germany.
}

Reduced glutathione (GSH) is an abundant low molecular weight plant thiol. It fulfills multiple functions in plant biology, many of which remain poorly characterized. A phenomics approach was therefore used to investigate the effects of glutathione homeostasis on growth and stress tolerance in Arabidopsis thaliana. Rosette leaf area was compared in mutants that are either defective in GSH synthesis (cad2, pad2, and rax1) or the export of $\gamma$-glutamylcysteine and GSH from the chloroplast $(c / t)$ and in wild-type plants under standard growth conditions and following exposure to a range of abiotic stress treatments, including oxidative stress, water stress, and high salt. In the absence of stress, the GSH synthesis mutants had a significantly lower leaf area than the wild type. Conversely, the $c / t$ mutant has a greater leaf area and a significantly reduced lateral root density than the wild type. These findings demonstrate that cellular glutathione homeostasis exerts an influence on root architecture and on rosette area. An impaired capacity to synthesize GSH or a specific depletion of the cytosolic GSH pool did not adversely affect leaf area in plants exposed to short-term abiotic stress. However, the negative effects of long-term exposure to oxidative stress and high salt on leaf area were less marked in the GSH synthesis mutants than the wild type. These findings demonstrate the importance of cellular glutathione homeostasis in the regulation of plant growth under optimal and stress conditions.

\section{Keywords: abiotic stress tolerance, glutathione synthesis, root architecture, lateral root density, leaf area}

\section{INTRODUCTION}

Environmental stresses severely limit plant growth and decrease the predictability of crop yields for the farmer. Abiotic stress often has a greater impact on crop productivity than genotypic effects. Enhancing stress tolerance is therefore a major secondgeneration trait target for crop improvement programs. Plant stress responses are complex traits regulated by large numbers of genes and quantitative trait loci (QTL). This complexity has restricted the success of conventional breeding approaches. Similarly, transgenic approaches to enhancing tolerance to complex stresses such as drought have not as yet significantly reduced environmentally related yield losses under field conditions (Lawlor, 2013). A greater understanding of the mechanisms that restrict the growth of plants in response to the imposition of abiotic stress is required to facilitate development and molecular breeding of crop varieties with enhanced stress tolerance traits.

Enhanced cellular oxidation is a common feature of the plant response to stress. Oxidative signaling underpins plant responses to stress and is intimately associated with hormone signaling pathways that regulate plant growth, senescence, and cell death responses. The thiol tripeptide, glutathione (GSH; $\gamma$-glutamyl-L-cysteinylglycine) is an important component of the plant antioxidant system that protects against the harmful effects of uncontrolled oxidation (Noctor and Foyer, 1998; Noctor et al., 2013). Moreover, GSH acts downstream of hydrogen peroxide in mediating the stress responses of phytohormones such as jasmonate and salicylate (Mhamdi et al., 2010). Many studies have implicated GSH in biotic and abiotic stress tolerance (Noctor and Foyer, 1998; Ogawa, 2005).

The pathway of GSH synthesis involves two ATP-dependent steps catalyzed by g-glutamate-cysteine ligase (GCL; also called $\gamma$-glutamylcysteine ( $\gamma$-EC) synthetase), which is considered to be the rate-limiting enzyme of GSH production, and GSH synthetase (GSHS; also called GSH synthase). In A. thaliana, the GCL protein is found only in chloroplasts and other plastids, whereas the GSH$\mathrm{S}$ is found in both the chloroplasts and cytosol (Wachter et al., 2005).

Plant homologs of the malaria chloroquine-resistance transporter PfCRT (CLTs) are thiol transporters required for transport of $\gamma$-EC and GSH across the plastid envelope membranes and interconnect the plastidic and cytosolic thiol pools (Maughan etal., 2010). Arabidopsis mutants lacking these transporters (clt1clt2clt3) show enhanced sensitivity to cadmium and to the fungal pathogen, Phytophthora brassicae, as well as a failure to activate appropriate pathogen defense responses despite having wild-type levels of GSH in the leaves (Maughan et al., 2010). The 
clt1clt2clt3 mutants have an altered partitioning of GSH between plastid and cytosol, with a marked decrease in the cytosolic GSH levels but not in the chloroplast GSH pool in the leaves (Maughan et al., 2010). The cytosolic GSH pool is important in the mediation of systemic acquired resistance responses linked to salicylic acid signaling, as demonstrated by defects in pathogen-resistance and the expression of pathogenesis-resistance protein (PR) 1 in the clt1clt2clt3 triple mutants (Maughan et al., 2010) and in mutants that are defective in the cytosolic/peroxisomal form of NADPHdependent glutathione reductase ( $g r 1)$. These mutants accumulate less salicylic acid with lower PR1 transcripts under oxidative stress conditions (Mhamdi et al., 2010). Mutants lacking the chloroplast/mitochondrial GR2 are embryo-lethal (Tzafrir et al., 2004) but the gr1 knockout mutants do not show a marked phenotype (Marty et al., 2009; Mhamdi et al., 2010). However, crossing the gr1 knockout mutants with a mutant that is defective in the photorespiratory form of catalase cat2, led to a large accumulation in GSSG relative to the parent lines (Mhamdi et al., 2010). The cat2 grl double mutants that are deficient in both the major leaf catalase isoform and GR1 have altered responses to pathogens and expression of genes involved in jasmonate and salicylate signaling pathways (Mhamdi et al., 2010).

Glutathione synthesis and accumulation are increased in response to oxidative stress (Queval et al., 2009) because of direct effects of oxidation on the GCL protein, which is most active in its homodimeric form requiring linkage through two disulfide bonds (Hothorn et al., 2006). Reducing conditions disrupt one of the two disulfide bonds (Cys178-Cys398) altering the dimer interface and shifting the protein to the less active monomeric form (Jez et al., 2004; Hothorn et al., 2006; Galant et al., 2011). Oxidation-dependent decreases in cellular GSH/GSSG ratios also favor increased synthesis of cysteine, which is also considered to be a limiting factor for GSH synthesis (Noctor et al., 2012).

Arabidopsis mutants, with defects in the pathway of GSH synthesis, have been particularly useful in the characterization of GSH functions in plants. A number of mutants with defects in the GSH1 gene that encodes GCL have been identified and these were often first characterized in terms of effects on stress tolerance. For example, the cad2-1 mutant that has 15-30\% of wild-type GSH was identified by its enhanced sensitivity to cadmium (Cobbett et al., 1998), the $\operatorname{rax1-1}$ mutant, where the leaf GSH pool is decreased by between 50 and $80 \%$ relative to the wild type, was identified by the altered expression of the gene encoding the cytosolic ascorbate peroxidase (Ball et al., 2004) and the pad2-1 mutant, where leaf GSH is decreased by $80 \%$ compared to the wild type, shows enhanced sensitivity to fungal pathogens such as $P$. brassicae and Pseudomonas syringae because of decreased camalexin content (Parisy et al., 2007). Mutations in the GSH2 gene that encodes GSHS have also been very useful in elucidating the functions of glutathione in plants (Pasternak et al., 2008; Au et al., 2012). The morphology of the endoplasmic reticulum is altered and protein export is perturbed when $\gamma$-glutamylcysteine accumulates as a result of limitations in GSHS activity (Au et al., 2012). This may explain why the levels of $\gamma$-glutamylcysteine are very low in plant cells (Noctor et al., 1998).

In addition, to its antioxidant and signaling functions, GSH is also required for plant growth and development. GSH deficiency leads to an arrest in cell proliferation and root meristem formation (Vernoux et al., 2000; Diaz-Vivancos et al., 2010). GSH also has roles in flower development and vernalization responses (Bashandy et al., 2010; Hatano-Iwasaki and Ogawa, 2012). Despite this, the role of GSH in the control of plant growth under abiotic stress conditions is largely unknown.

Phenomics technologies allow accurate measurements of leaf area in large numbers of plants grown in either the absence or presence of abiotic stress over periods of days to weeks. This approach was therefore used to explore the effects of GSH deficiency on rosette leaf area in different GSH synthesis mutants (cad2-1, rax11 , and pad2-1) and in the clt1clt2clt3 triple mutants, which have altered intracellular partitioning of GSH between the chloroplasts and cytosol, under either standard (optimal) growth conditions and under abiotic stress conditions. The findings show that in contrast to the GSH synthesis mutants, which have a lower leaf area in the absence of stress, leaf area was increased in the clt1clt 2 clt 3 triple mutants relative to wild-type controls. The abiotic stress-induced decreases in leaf area were similar in all genotypes in short-term experiments. However, in the longer term stress treatments, the negative impacts of some abiotic stresses on leaf area were less marked in the GSH synthesis mutants than in the wild-type plants or in the clt1clt2clt3 mutants.

\section{MATERIALS AND METHODS PLANT MATERIAL}

Seeds for wild-type A. thaliana accession Columbia 0 (Col-0), cad2-1 (Cobbett et al., 1998), pad2-1 (Parisy et al., 2007), rax1-1 (Ball et al., 2004), rml1-1 (Cheng et al., 1995; Vernoux et al., 2000), and clt1clt2clt3 triple mutants (Maughan et al., 2010), were sown on plates containing half strength Murashige and Skoog medium plus $1.2 \%$ glucose. Plants were grown in controlled environmental cabinets under an irradiance of $100 \mu \mathrm{mol} \mathrm{m}^{-2} \mathrm{~s}^{-1}$ with a photoperiod of $16 \mathrm{~h}$, a constant temperature of $22 \pm 2^{\circ} \mathrm{C}$, and a relative humidity of $60 \%$, for up to 17 days. For high light treatments, seedlings were grown for 10 days as above and then transferred to $400 \mu \mathrm{mol} \mathrm{m}{ }^{-2} \mathrm{~s}^{-2}$ irradiance conditions for a further 4 days. The seedlings were then transferred back to $100 \mu \mathrm{mol} \mathrm{m}^{-2} \mathrm{~s}^{-1}$ for a further 3 days. Each experiment consisted of four plates (32 seeds per plate) per genotype and per stress treatment. Each experiment was repeated at least three times.

\section{SHORT STRESS TREATMENTS}

For these experiments, seeds were sown on a sterile $1 \mu \mathrm{m}$ filter mesh, which was placed on the media prior to sowing. Seedlings were grown for 10 days under the $100 \mu \mathrm{mol} \mathrm{m}^{-2} \mathrm{~s}^{-1}$ irradiance conditions and then transferred with the mesh to plates containing media alone (control) or growth media plus either hydrogen peroxide $(4 \mathrm{mM})$, sodium chloride $(75 \mathrm{mM}), N, N$ '-dimethyl4,4'-bipyridinium dichloride (paraquat; $1 \mu \mathrm{M}$ ), or sorbitol (100 mM). Seedlings were then grown for a further 7 days under these conditions and growth analysis performed as described below.

\section{LONG STRESS TREATMENTS}

For these experiments, seeds were sown on plates in media alone (control), or media containing paraquat $(0.1 \mu \mathrm{M})$, sodium 
chloride (75 mM), or sorbitol (100 mM). Seedlings were grown for 14 days under these conditions. Lower concentrations of paraquat were used in the long-term stress treatments than in the short-term stress treatments because preliminary experiments had shown that the higher concentrations of paraquat were lethal in long-term experiments. In contrast, the lower concentrations of paraquat had little effect on leaf area in the short-term experiments.

\section{LEAF AREA DETERMINATION}

Leaf area measurements were performed as described by Schulz etal. (2012). Photographs were taken with a Canon EOS 450 D (Canon Inc., Tokyo, Japan) on successive days after sowing. Data presented here are taken from measurements made 14 and 17 days after sowing only. Total rosette surface area (hereafter called leaf area) was measured and analyzed using Fiji Image ${ }^{1}$ as described by Schindelin et al. (2012). Data was processed using Microsoft Excel 2010 and statistical analysis was performed with program $\mathrm{R}^{2}$ (Hornik, 2013). The ImageJ analysis used here to calculate leaf area measures the total rosette (leaf) surface, which is highly correlated with the fresh weigh of the plant (usually $r=0.9-0.95)$.

\section{ROOT GROWTH AND ROOT ARCHITECTURE DETERMINATION}

Primary root length and number of lateral roots were measured on 7-day-old seedlings. Root measurements were determined using Fiji ImageJ software, as above. Lateral root density was calculated from these values as the ratio between the number of visible lateral roots and the primary root length.

\section{METABOLITE ANALYSIS}

For these experiments, the wild-type and clt1clt2clt3 plants were grown in pots containing compost (Levington, Bramford, UK) in controlled environment chambers $(16 / 8 \mathrm{~h}$ light/dark regime with photosynthetic photon flux of $\left.250 \mu \mathrm{mol} \mathrm{m} \mathrm{m}^{-2} \mathrm{~s}^{-1}\right)$. The whole rosettes of 5-week-old plants were harvested and assayed for ascorbate, glutathione, and pyridine nucleotides as described by Pellny et al. (2009).

\section{RESULTS}

The open-source platform for biological-image analysis used in these studies involves ImageJ analysis measurements of the total rosette (leaf) surface area (from hereon called leaf area), a parameter which is highly correlated to the fresh weigh of the plant (usually $r=0.9-0.95)$. Total leaf surface area was compared in rax1-1, cad2-1, and pad2-1 mutants with that of wild-type $A$. thaliana seedlings at 14 days (Figure 1). The genotypes that are deficient in GSH synthesis were visibility smaller (Figure 1A) and they had a significantly lower leaf area than the wild type (Figure 1B).

The effects of short-term (7 days) exposure to different abiotic stress treatments on leaf area was measured (Figure 2). Exposure to oxidative stress ( $4 \mathrm{mM}$ hydrogen peroxide or $1 \mu \mathrm{M}$ paraquat), high salt ( $75 \mathrm{mM}$ sodium chloride), or osmotic stress ( $100 \mathrm{mM}$ sorbitol) led to a visible shoot phenotype (Figure 2A) and a decrease in leaf area in all genotypes (Figures 2B-E). All treatments except

\footnotetext{
${ }^{1}$ http://fiji.sc/

${ }^{2}$ www.r-project.org
}

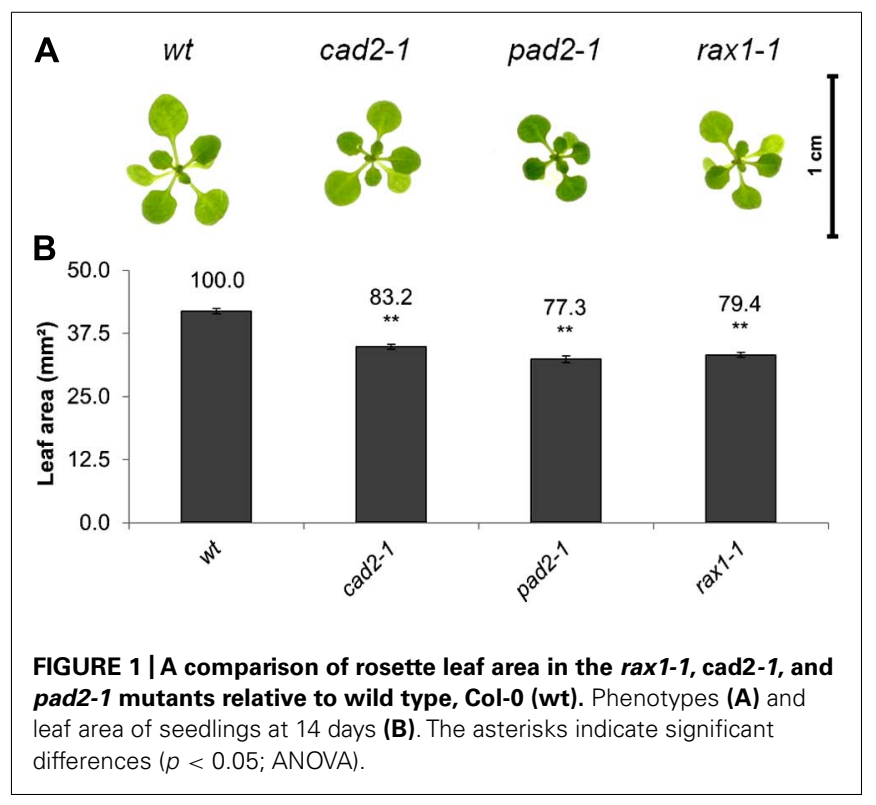

exposure to hydrogen peroxide led to a significant decrease in leaf area in all genotypes (Figures 2B-E). The genotypes that were defective in GSH synthesis were not more sensitive to the treatments in relation to leaf area than the wild type except for the $\operatorname{rax} 1-1$, which was more sensitive to the paraquat treatment (Figure 2E). However, the pad2-1 mutants were less sensitive to the paraquat treatment relative to the wild type (Figure 2D). The cad2-1 and pad2-1 mutants were also less sensitive to the high salt treatment than the wild type (Figure 2C). Short-term (4 days) exposure to a relatively high light $\left(400 \mu \mathrm{mol} \mathrm{m}{ }^{-2} \mathrm{~s}^{-1}\right)$ treatment visibly stimulated increases in leaf area (Figure $2 \mathbf{A}$ ) and led to a significant increase in leaf area in all genotypes (Figure 2F). Moreover, under these conditions the pad2-1 mutants had a similar leaf area to the wild type, whereas the leaf area in the cad2-1 and rax1-1 plants was significantly smaller than the wild type (Figure 2F).

The effects of long-term (14 days) exposure to the different abiotic stress treatments such as oxidative stress (paraquat), salt stress (sodium chloride), or osmotic stress (sorbitol) on leaf area was measured in all genotypes (Figure 3). All treatments led to a visible decrease in the rosettes of all genotypes (Figure 3A) and significant decrease in leaf area in all cases (Figures 3B-E). In contrast to the osmotic stress treatment, which led to similar decrease in leaf area in all genotypes except the pad2-1 mutants, the GSH synthesis mutants had a significantly greater leaf area than the wild type in the oxidative stress treatment (paraquat) and in the high salt treatment (Figures 3B-E).

The ascorbate and glutathione contents of the clt1clt2clt3 leaves were similar to that of the wild type (Table 1). The rosettes of the clt1clt2clt3 triple mutants were visibly larger than those of the wild type (Figure 4A) and they had significantly greater leaf area (Figure 4B). However, root architecture was markedly different in the clt1clt2clt3 triple mutants relative to the wild type (Figure 5A). The primary root length was significantly shorter than that of the wild type (Figure 5B) and there were significantly fewer lateral roots (Figure 5C). The lateral root density was 

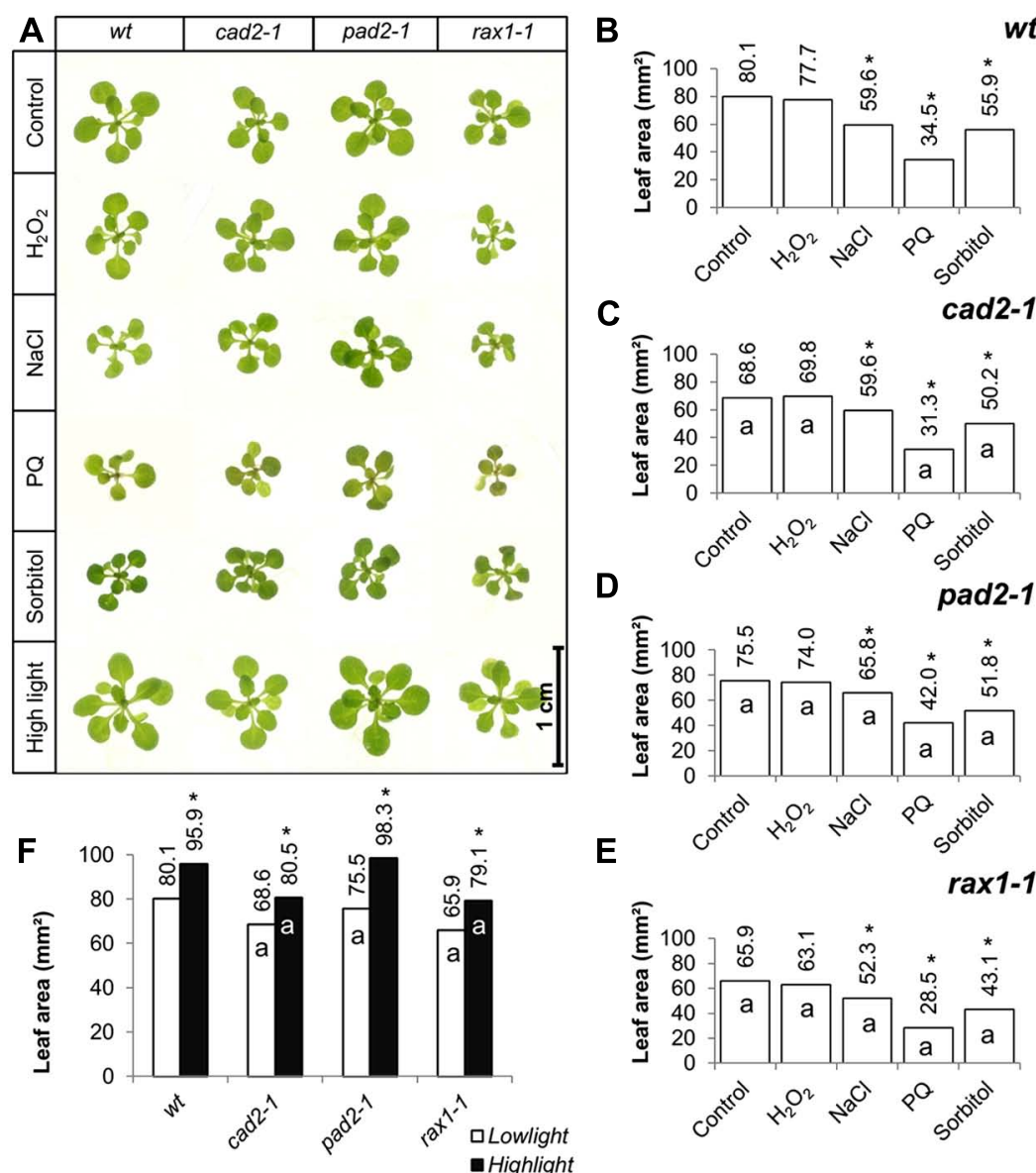

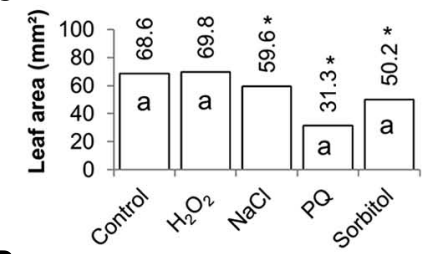

D pad2-1
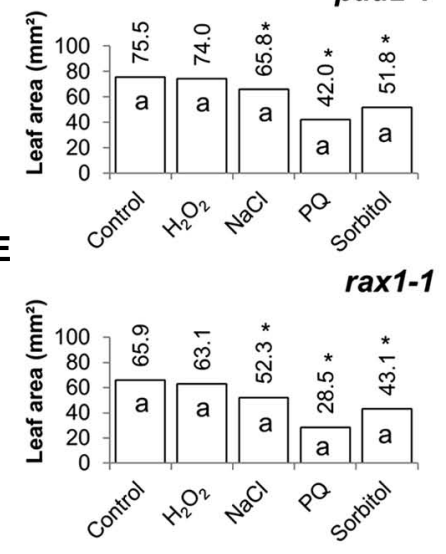

FIGURE 2 | A comparison of the effects of short-term (4 days) exposures to different abiotic stresses treatments on rosette leaf area in the rax 1-1, cad2-1, and pad2-1 mutants relative to wild type, Col-0 (wt). In all cases seedlings were grown for 10 days under optimal conditions and then transferred to different abiotic stress treatments for a further 4 days. Phenotypes (A) and leaf area (B-F) of seedlings measured at 14 days either in the absence (control) or presence of the oxidative stress caused by the addition of hydrogen peroxide $\left(\mathrm{H}_{2} \mathrm{O}_{2}\right)$ or paraquat (PQ), or high salt (sodium chloride, $\mathrm{NaCl}$ ), or osmotic stress (sorbitol). For the high light treatment [closed bars, (F)] seedlings were either grown under $150 \mu \mathrm{mol} \mathrm{m}^{-2} \mathrm{~s}^{-1}$ irradiance for 14 days (open columns) or they were grown under $150 \mu \mathrm{mol} \mathrm{m} \mathrm{m}^{-2} \mathrm{~s}^{-1}$ irradiance for 10 days and then transferred to $400 \mu \mathrm{mol} \mathrm{m}{ }^{-2} \mathrm{~s}^{-1}$ irradiance for 4 days prior to measurement. The asterisks indicate significant differences ( $p<0.05$; ANOVA). markedly decreased in the $c l t 1 c l t 2 c l t 3$ triple mutants relative to the wild type (Figure 5D).

The effects of short-term (7 days) exposure to abiotic stress on leaf area were compared in the clt1clt2clt3 triple mutants and wild-type plants (Figure 6A). Exposure to low levels of hydrogen peroxide $(4 \mathrm{mM})$ had no effect on leaf area in either genotype (Figure 6). Salt stress caused a significant decrease in leaf area in both genotypes, but the salt-induced decrease in leaf area was greater in the wild type than the clt1clt2clt3 triple mutants (Figures 6B,C). Similarly, exposure to paraquat caused a significant decrease in leaf area in both genotypes, but the oxidative stress-induced decrease in leaf area was greater in the wild type than the clt1clt2clt3 triple mutants (Figures 6B,C). Moreover, exposure to osmotic stress caused a significant decrease in leaf area in both genotypes, but the osmotic stress-induced decrease in leaf area was greater in the wild type than the clt1clt2clt3 triple mutants (Figures 6B,C). Short-term (4 days) exposure to a high light resulted in visibly larger rosettes (Figure 6A) and led to a significant increase in leaf area in all genotypes (Figure 6D).

The effects of long-term (14 days) exposure to the different abiotic stress treatments were compared in the clt1clt 2 clt 3 triple mutants and wild-type plants (Figure 7A). All treatments led to a visible decrease in the size of the rosettes of both genotypes (Figure 7A). A significant decrease in leaf area was observed following exposure to oxidative stress, high salt, and osmotic stress in both genotypes (Figures 7B,C).

The total leaf ascorbate or glutathione contents were similar in the clt1clt2clt3 triple mutants and the wild type (Table 1). While the $\mathrm{NAD}^{+} / \mathrm{NADH}$ ratios were also similar in the leaves of both genotypes, the clt1clt2clt3 triple mutant leaves had higher $\mathrm{NADP}^{+} / \mathrm{NADPH}$ ratios than those of the wild type (Table $\mathbf{1}$ ).

\section{DISCUSSION}

Genetic evidence has demonstrated links between glutathione redox state and shoot and root meristem activity (Vernoux et al., 


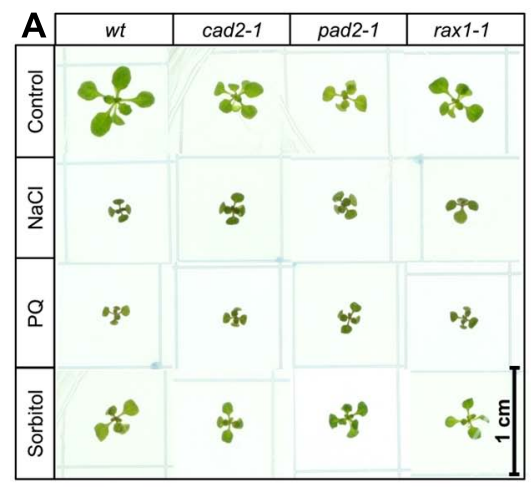

E

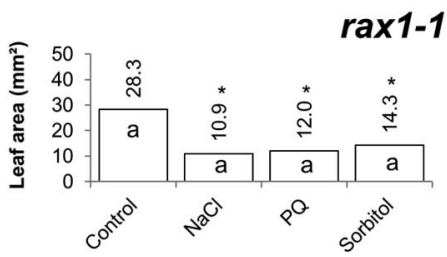

FIGURE 3 | A comparison of the effects of long-term (14 days) exposures to different abiotic stresses treatments on rosette leaf area in the rax 1-1, cad2-1, and pad2-1 mutants relative to wild type, Col-0 (wt). Phenotypes (A) and leaf area (B-E) of seedlings measured at 14 days either in the

Table 1 | A comparison of the major redox metabolites in the rosette leaves of the clt1clt2clt3 triple mutants and wild-type (Col-0) plants.

\begin{tabular}{|c|c|c|}
\hline \multirow[t]{2}{*}{ Metabolite } & \multicolumn{2}{|c|}{ Genotype } \\
\hline & Col-0 & clt1clt2clt3 \\
\hline Ascorbate ( $\left.\mu \mathrm{mol} \mathrm{mg} \mathrm{m}^{-1} \mathrm{Chl}\right)$ & $3.48 \pm 0.17^{a}$ & $4.20 \pm 0.18^{a}$ \\
\hline Dehydroascorbate $\left(\mu \mathrm{mol} \mathrm{mg}^{-1} \mathrm{Chl}\right)$ & $1.34 \pm 0.14^{\mathrm{a}}$ & $1.01 \pm 0.16^{\mathrm{a}}$ \\
\hline Ascorbate/dehydroascorbate & $2.59^{a}$ & $3.82^{\mathrm{a}}$ \\
\hline $\mathrm{GSH}\left(\mu \mathrm{mol} \mathrm{mg}^{-1} \mathrm{Chl}\right)$ & $228.33 \pm 30.10^{a}$ & $264.67 \pm 30.64^{\circ}$ \\
\hline GSSG $\left(\mu \mathrm{mol} \mathrm{mg}{ }^{-1} \mathrm{Chl}\right)$ & $7.51 \pm 0.46^{a}$ & $10.73 \pm 0.81^{\mathrm{a}}$ \\
\hline GSH/GSSG & $31.23^{\mathrm{a}}$ & $20.10^{\mathrm{a}}$ \\
\hline $\mathrm{NADH}\left(\mu \mathrm{mol} \mathrm{mg}{ }^{-1} \mathrm{Chl}\right)$ & $2.73 \pm 0.48^{a}$ & $2.44 \pm 0.16^{a}$ \\
\hline $\mathrm{NAD}\left(\mu \mathrm{mol} \mathrm{mg}{ }^{-1} \mathrm{Chl}\right)$ & $7.12 \pm 1.12^{\mathrm{a}}$ & $5.61 \pm 0.77^{a}$ \\
\hline NADH/NAD & $0.38^{a}$ & $0.43^{a}$ \\
\hline $\mathrm{NADPH}\left(\mu \mathrm{mol} \mathrm{mg}{ }^{-1} \mathrm{Chl}\right)$ & $25.59 \pm 5.42^{a}$ & $16.45 \pm 2.50^{\mathrm{b}}$ \\
\hline NADP $\left(\mu \mathrm{mol} \mathrm{mg}^{-1} \mathrm{Chl}\right)$ & $1.77 .88 \pm 0.09^{a}$ & $4.22 \pm 0.40^{\mathrm{b}}$ \\
\hline NADPH/NADP & $15.02^{\mathrm{a}}$ & $3.90^{\mathrm{b}}$ \\
\hline
\end{tabular}

Values represent the mean values $\pm S E(n=6)$. Values within a single row that were significantly different as estimated by Fishers protected LSD test $(p<0.05)$ are indicated by different letters.

2000; Reichheld et al., 2007; Bashandy et al., 2010; Koprivova et al., 2010). However, mechanisms by which GSH participate in the control of growth particularly under stress conditions remain to be characterized. The rml1-1 mutant, which has less than $5 \%$ of the wild-type GSH levels is unable to establish a post-embryonic root meristem because of cell cycle arrest at G1 (Vernoux et al.,

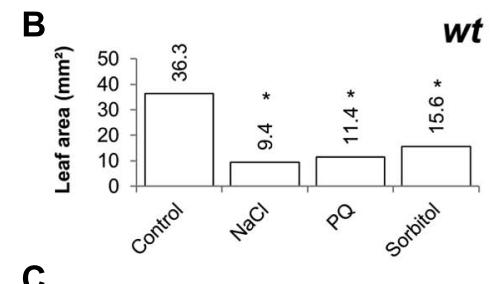

C
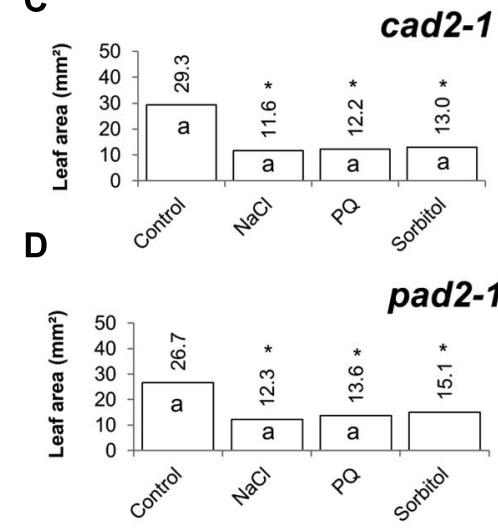

absence (control) or presence of the oxidative stress caused by the addition of paraquat (PQ) or high salt (sodium chloride, $\mathrm{NaCl}$ ), or osmotic stress (sorbitol). The asterisks indicate significant differences $(p<0.05$; ANOVA).

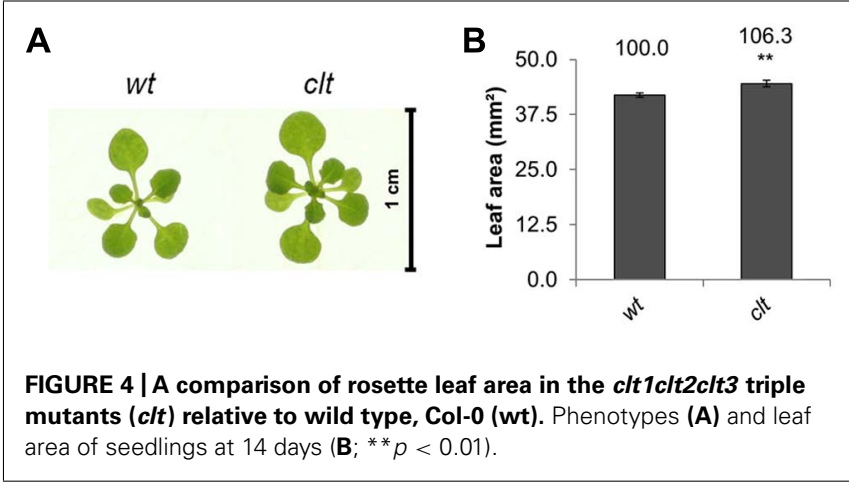

2000). Similarly, a pharmacological approach has demonstrated that inhibition of GSH synthesis also leads to an arrest of root growth (Koprivova et al., 2010). Under similar growth conditions to those used here the cad2-1, pad2-1, and rax1-1 mutants had a lower number of lateral roots leading to a lower lateral root density in all the GSH deficient mutant genotypes compared to the wild type (Marquez-Garcia et al., 2013). While visual inspection failed to establish a clear phenotype under routine growth conditions (Maughan et al., 2010), the results presented here clearly demonstrate that like the GSH synthesis mutants, the clt1clt2clt3 triple mutants have significantly lower lateral root densities than the wild type. These data indicate that a high cytosolic GSH pool is required in the control of root architecture. Root growth may be regulated in part by the shoot; the decrease in the cytosolic GSH levels in clt1clt2clt3 leaves leading to GSH depletion in the roots, which rely, at least in part, on GSH transport from the 

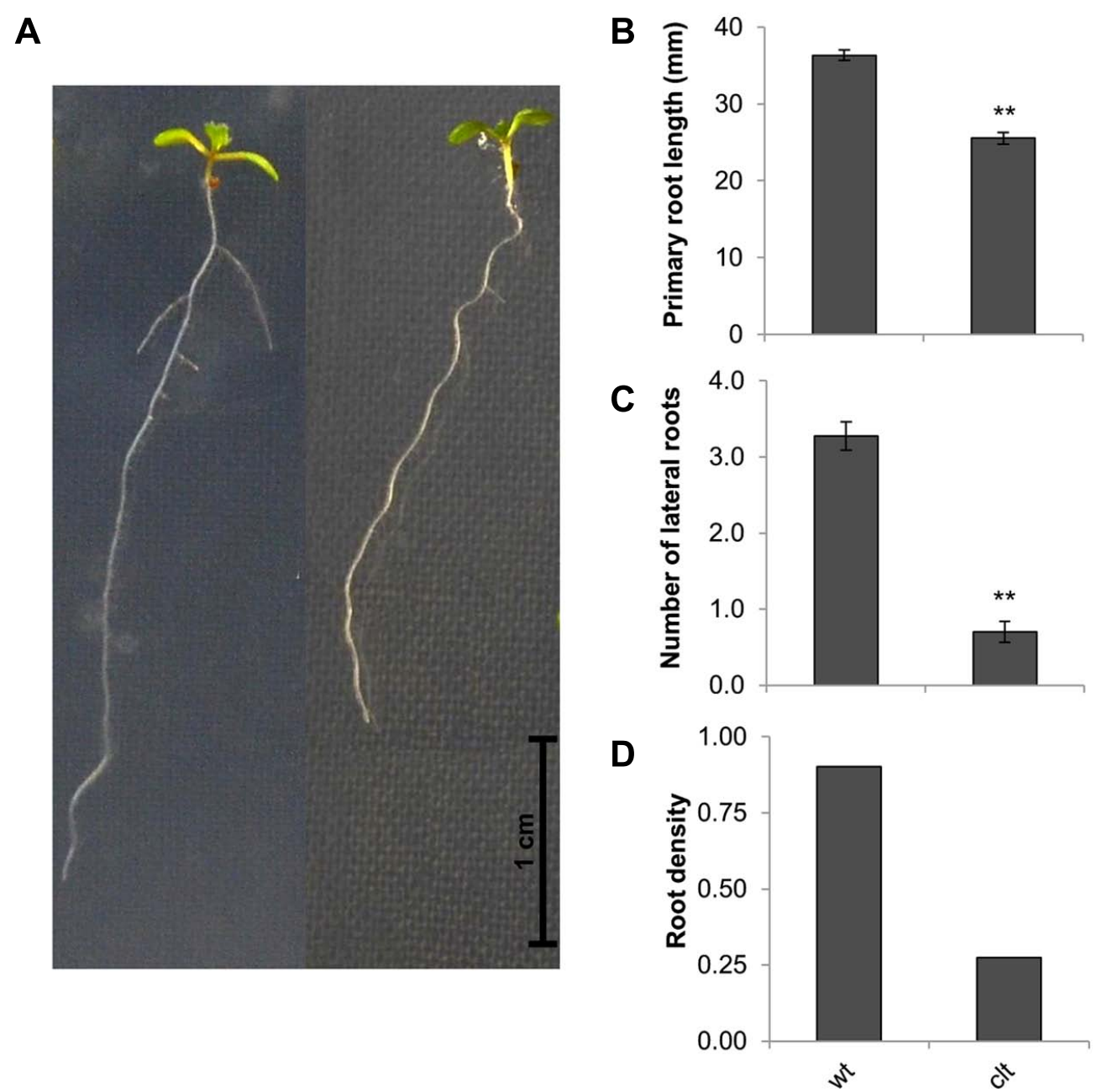

FIGURE 5 | Root architecture the clt1clt2clt3 triple mutants (clt) relative to wild type Col-0 (wt). The phenotype of 14-day-old seedlings (A), primary root length (B), number of visible lateral roots (C), and lateral root density (D). The asterisks indicate significant differences ( $p<0.05$; ANOVA).

leaves (Noctor et al., 1998; Li et al., 2006). Moreover, whereas the cad2-1, rax 1-1, and the pad2-1 mutants have decreased leaf area relative to the wild type, leaf area shows a small but significant increase in the clt1clt2clt3 triple mutants compared to the wild type. These data may implicate chloroplast GSH pool in the regulation of leaf area. However, rosette size was significantly enhanced by high light, which led to a significant increase in leaf area in all genotypes. Under these conditions the pad2-1 mutants performed better than the cad2-1 and rax1-1 mutants, achieving leaf areas that were similar to or even slightly higher than the wild type, suggesting that high light can overcome the adverse influence of low GSH in signaling that controls leaf area.

The results presented here show that the leaves of the clt1clt2clt3 mutants had a similar redox status to the wild-type plants, under optimal growth conditions, with comparable ascorbate and glutathione levels and similar ascorbate/dehydroascorbate, GSH/GSSG, and NAD/NADH ratios, even though the partitioning of GSH between the cytosol and chloroplasts was changed in the clt1clt2clt3 mutants relative to the wild type (Maughan et al., 2010).
The observed decreases in the leaf $\mathrm{NADPH} / \mathrm{NADP}^{+}$ratios in the clt1clt2clt 3 mutants linked to the altered intracellular partitioning of GSH between these compartments, might be explained by in terms of increased demand for NADPH for cytosolic redox processes linked to thioredoxin, as a result of GSH depletion (Marty et al., 2009).

Short-term exposures to oxidative stress (paraquat), salt stress, and osmotic stress resulted in a decrease in leaf area in all genotypes. However, the stress effects were similar in the GSH synthesis mutants and in the clt1clt2clt3 triple mutants to the wild type, the only exception being the rax 1-1 mutant, which was slightly more sensitive to the paraquat treatment. Longer term abiotic stress treatments caused larger decreases in leaf area in all genotypes. However, in contrast to the clt1clt2clt 3 triple mutants, which showed a similar response to the abiotic stresses to the wild-type plants, the GSH synthesis mutants with the exception of pad2-1, had a significantly greater leaf area than the wild type under the oxidative stress and the high salt treatments. These results suggest that impaired GSH synthesis capacity may therefore serve to 


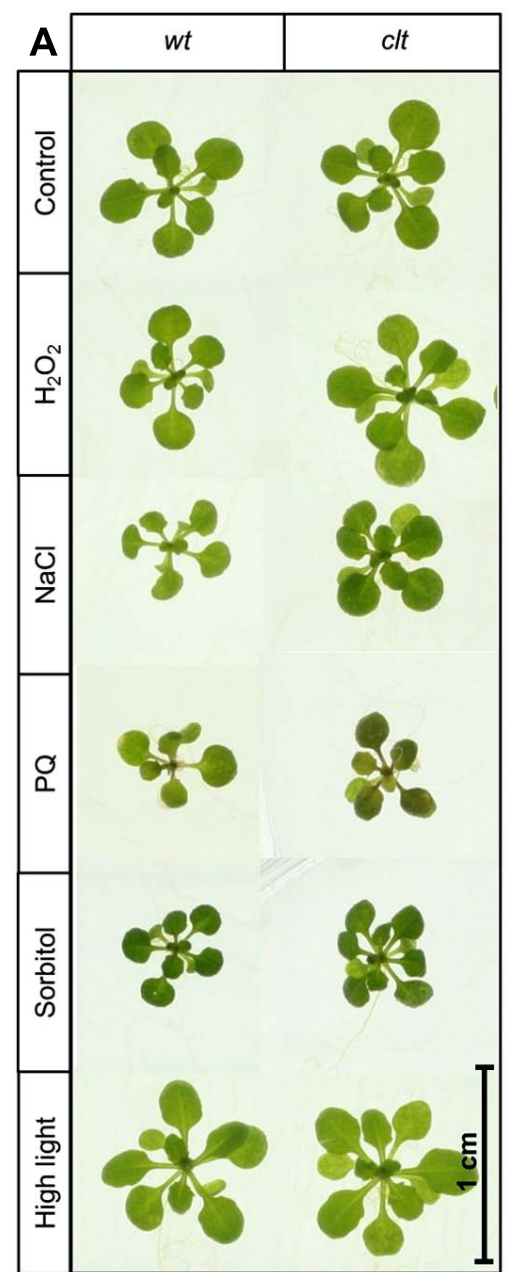

FIGURE 6 | A comparison of the effects of short-term (4 days) exposures to different abiotic stresses treatments on rosette leaf area in the clt1clt2clt3 triple mutants relative to wild type, Col-0 (wt). In (A-C) seedlings were grown for 10 days under optimal conditions and then transferred different abiotic stress treatments for a further 4 days.

Phenotypes $\mathbf{( A )}$ and leaf area (B,C) of seedlings measured at 14 days either in the absence (control) or presence of the oxidative stress caused by the
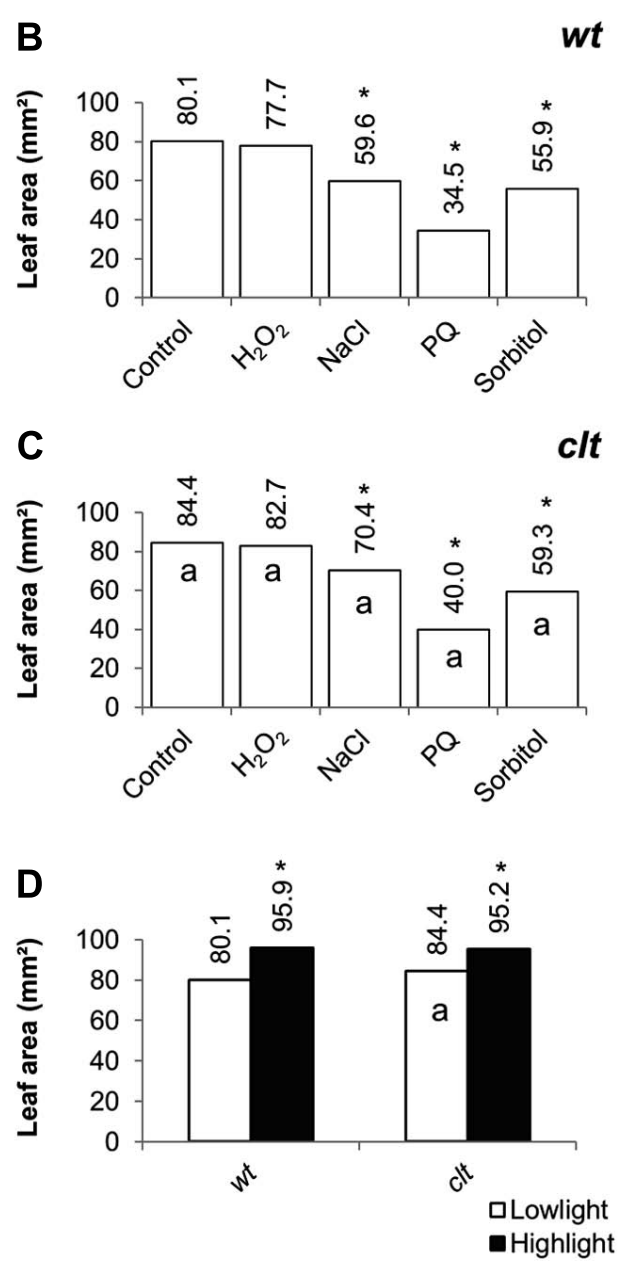

addition of hydrogen peroxide $\left(\mathrm{H}_{2} \mathrm{O}_{2}\right)$ or paraquat (PQ), or high salt (sodium chloride, $\mathrm{NaCl}$ ), or osmotic stress (sorbitol). For the high light treatment (closed bars, (D)) seedlings were either grown under $150 \mu \mathrm{mol} \mathrm{m} \mathrm{m}^{-2} \mathrm{~s}^{-1}$ irradiance for 14 days (open columns) or they were grown under $150 \mu \mathrm{mol} \mathrm{m}-2 \mathrm{~s}^{-1}$ irradiance for 10 days and then transferred to $400 \mu \mathrm{mol} \mathrm{m}{ }^{-2} \mathrm{~s}^{-1}$ irradiance for 4 days prior to measurement. The asterisks indicate significant differences ( $p<0.05$; ANOVA). mitigate the adverse effects of some abiotic stresses such as salt stress and dehydration on leaf growth but not others such as heavy metal stress, where GSH is required for other pathways such as phytochelatin biosynthesis.

The observation that GSH deficiency limits the adverse effects of salt stress and dehydration on leaf growth may be related to the central role of glutathione in the regulation of gene expression linked to oxidative stress signaling (Noctor et al., 2013). Accumulating evidence supports the concept that glutathione status is involved in the cross talk between oxidative signaling and hormone signaling (Mhamdi et al., 2010; Han et al., 2013a,b). Crucially, glutathione status also influences the auxin signaling pathways that control growth (Bashandy et al., 2010; Gao et al., 2013). The oxidative signals that limit growth under oxidative stress are therefore likely to be transmitted at least in part via modulation of the redox status of the glutathione pool. The greater leaf area observed here in the GSH synthesis mutants relative to the wild type under oxidative and high salt stresses may therefore be linked to a requirement for GSH in the cross talk between redox and hormone-mediated signaling processes that serve to restrict growth in plants exposed to abiotic stress.

\section{CONCLUSION}

Abiotic stress tolerance is an important factor determining plant growth and productivity, and is the subject of ever-intensifying interest in relation to crop improvement. The importance of antioxidants such as glutathione in abiotic stress tolerance is well documented, particularly with regard to its antioxidant functions in protection against stress-induced oxidation. In addition to its potential usefulness as a stress marker, glutathione status is 


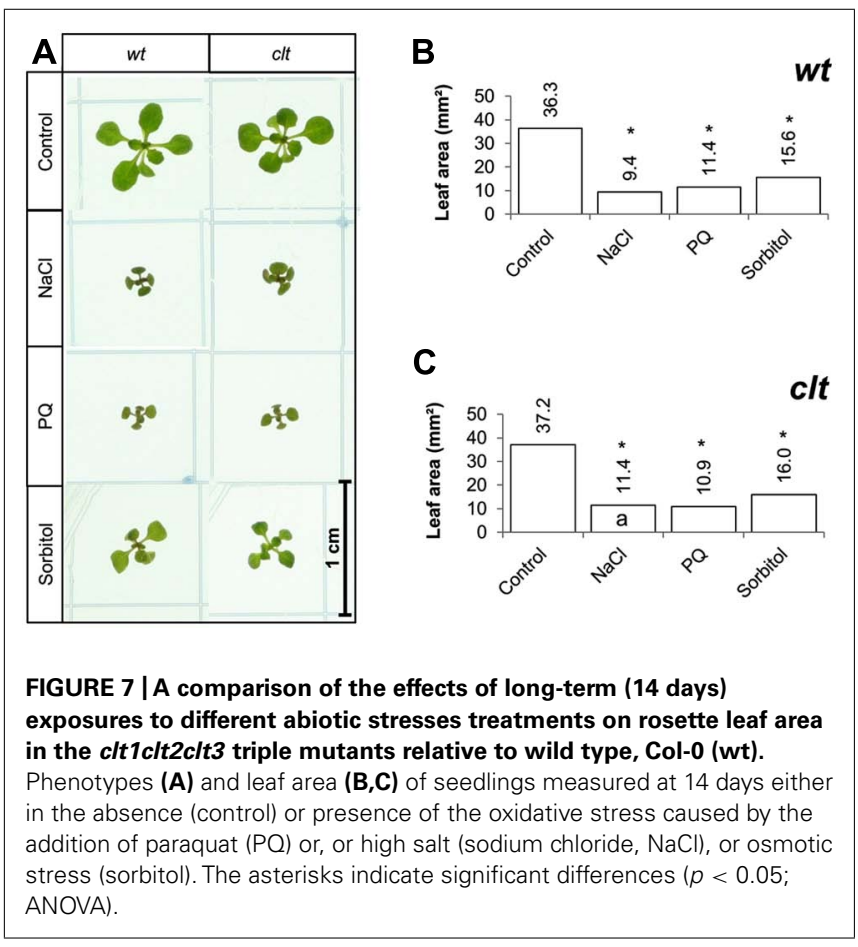

important in the control of growth and oxidative stress signaling (Noctor et al., 2013). Within this context, the results presented here demonstrates that the intracellular compartmentalization

\section{REFERENCES}

Au, K. K. C., Perez-Gomez, J., Neto, H., Muller, C., Meyer, A., Fricker, M. D., etal. (2012). A perturbation in glutathione biosynthesis disrupts endoplasmic reticulum morphology and secretory membrane traffic in Arabidopsis thaliana. Plant J. 71, 881-894. doi: 10.1111/j.1365313X.2012.05022.x

Ball, L., Accotto, G., Bechtold, U., Creissen, G., Funck, D., Jimenez, A., et al. (2004). Evidence for a direct link between glutathione biosynthesis and stress defense gene expression in Arabidopsis. Plant Cell 16, 2448-2462. doi: $10.1105 /$ tpc. 104.022608

Bashandy, T., Guilleminot, J., Vernoux, T., Caparros-Ruiz, D., Ljung, K., Meyer, Y., et al. (2010). Interplay between the NADP-linked thioredoxin and glutathione systems in Arabidopsis auxin signaling. Plant Cell 22, 376-339. doi: 10.1105/tpc.109.071225

Cheng, J. C., Seeley, K. A., and Sung, Z. R. (1995). RML1 and RML2, Arabidopsis genes required for cell proliferation at the root tip. Plant Physiol. 107, 365-376. doi: 10.1104/pp.107.2.365

Cobbett, C. S., May, M. J., Howden, R., and Rolls, B. (1998). The glutathione- deficient, cadmium-sensitive mutant, cad2-1, of Arabidopsis thaliana is deficient in gamma-glutamylcysteine synthetase. Plant J. 16, 73-78. doi: 10.1046/j.1365-313x.1998.00262.x

Diaz-Vivancos, P., Dong, Y. P., Ziegler, K., Markovic, J., Pallardó, F., Pellny, glutathione into the nucleus during cell proliferation adjusts whole cell redox homeostasis in Arabidopsis thaliana and lowers the oxidative defence shield. Plant J. 64, 825-838. doi: $\quad$ 10.1111/j.1365-313X.2010. 04371.x

Galant, A., Preuss, M. L., Cameron, J. C., and Jez, J. M. (2011). Plant glutathione biosynthesis: diversity in biochemical regulation and reaction products. Front. Plant Sci. 2:45. doi: 10.3389/fpls.2011.00045

Gao, X., Yuan, H.-M., Hu, Y.-Q., Li, J., and Lu, Y.-T. (2013). Mutation of Arabidopsis CATALASE2 results in hyponastic leaves by changes of auxin levels. Plant Cell Environ. doi: 10.1111/pce.12144 [Epub ahead of print].

Han, Y., Chaouch, S., Mhamdi, A., Queval, G., Zechmann, B., and Noctor, G. (2013a). Functional analysis of Arabidopsis mutants points to novel roles for glutathione in coupling $\mathrm{H}_{2} \mathrm{O}_{2}$ T. K., et al. (2010). Recruitment of

of glutathione influences plant growth, a depletion in the cytosol in the clt1clt2clt3 triple mutants leading to significant decreases in lateral root density and increases in rosette leaf area under nonstressed conditions. However, in contrast to biotic stress tolerance, which is impaired in the clt1clt2clt3 triple mutants (Maughan et al., 2010), depletion of the cytosolic GSH pool had no effect on the stress-induced decreases in leaf area in plants experiencing short or long periods of abiotic stress. Conversely, while decreases in GSH synthesis capacity resulted in significant decreases in lateral root density (Marquez-Garcia et al., 2013), this change in root architecture was accompanied by decreased rosette leaf area under non-stressed conditions. Moreover, limitations on GSH synthesis capacity favored larger leaf areas in plants experiencing long (but not short) periods of abiotic stress. Taken together, these findings shed new light on the functions of glutathione in plant growth and abiotic stress tolerance, showing that unexpectedly limitations on GSH synthesis enhance abiotic stress tolerance in the longer term as determined by leaf area. Moreover, while the intracellular partitioning of glutathione is important in the regulation of root architecture, it has little impact on leaf area and hence abiotic stress tolerance.

\section{ACKNOWLEDGMENTS}

This work was funded by FP7-PIRSES-GA-2008-230830 (LEGIM, Rosita E. Yocgo) and PITN-GA-2008-215174 (Chloroplast Signals, Daniel Schnaubelt). We thank J. P. Reichheld for the gift of $r m l 1$ mutant seed, P. Mullineaux for seeds of the rax1-1 mutants, and C. Cobbet for seeds of the triple $c l t$ and cad2-1 mutants.

to activation of salicylic acid accumulation and signaling. Antioxid. Redox Signal. 18, 2106-2121. doi: 10.1089/ars.2012.5052

Han, Y., Mhamdi, A., Chaouch, S. and Noctor, G. (2013b). Regulation of basal and oxidative stresstriggered jasmonic acid-related gene expression by glutathione. Plant Cell Environ. 36, 1135-1146. doi: 10.1111/pce. 12048

Hatano-Iwasaki, A., and Ogawa, K. (2012). Overexpression of the GSH1 gene mimics transcriptional response to low temperature during seed vernalization treatment of Arabidopsis. Plant Cell Physiol. 53, 1195-1203. doi: 10.1093/pcp/pcs075

Hornik, K. (2013). The $R$ FAQ. Available at: http://CRAN.Rproject.org/doc/FAQ/R-FAQ.html doi: $10.1111 /$ pce. 12144

Hothorn, M., Wachter, A., Gromes, R., Stuwe, T., Rausch, T., and Scheffzek, K. (2006). Structural basis for the redox control of plant glutamate cysteine ligase. J. Biol. Chem. 281, 27557-27565. doi: 10.1074/jbc.M602770200

Jez, J. M., Cahoon, R. E., and Chen, S. (2004). Arabidopsis thaliana glutamate-cysteine ligase: functional properties, kinetic mechanism, and regulation of activity. J. Biol. Chem. 279, 33463-33479. doi: 10.1074/jbc.M405127200

Koprivova, A., Mugford, S. T., and Kopriva, S. (2010). Arabidopsis root growth dependence on glutathione is linked to auxin transport. Plant Cell Rep. 29, 1157-1167. doi: 10.1007/s00299-010-0902-0

Lawlor, D. W. (2013). Genetic engineering to improve plant performance under drought: physiological evaluation of achievements, limitations, and possibilities. J. Exp. Bot. 64, 83-108. doi: 10.1093/ jxb/ers326

Li, Y., Dankher, O. P., Carreira, L., Smith, A. P., and Meagher, R. B. (2006). The shoot-specific expression of $\gamma$ glutamylcysteine synthetase directs the long-distance transport of thiolpeptides to roots conferring tolerance to mercury and arsenic. Plant Physiol. 141, 288-298. doi: 10.1104/pp.105. 074815

Marquez-Garcia, B., Njo, M., Beeckman, T., Goormachtig, S., and Foyer, C. H. (2013). A new role for glutathione in the regulation of root architecture linked to strigolactones. Plant Cell Environ. doi: 10.1111/pce.12172 [Epub ahead of print]. 
Marty, L., Siala, W., Schwarzländer, M., Fricker, M. D., Wirtz, M., Sweetlove, L. J., et al. (2009). The NADPH-dependent thioredoxin system constitutes a functional backup for cytosolic glutathione reductase in Arabidopsis. Proc. Natl. Acad. Sci. U.S.A. 106, 9109-9114. doi: 10.1073/pnas.0900206106

Maughan, S. C., Pasternak, M., Cairns, N., Kiddle, G., Brach, T., Jarvis, R., et al. (2010). Plant homologs of the Plasmodium falciparum chloroquineresistance transporter, PfCRT, are required for glutathione homeostasis and stress responses. Proc. Natl. Acad. Sci. U.S.A. 107, 2331-2336. doi: 10.1073/pnas.0913689107

Mhamdi, A., Hager, J., Chaouch, S., Queval, G., Han, Y., Taconnat, L., et al. (2010). Arabidopsis GLUTATHIONE REDUCTASE 1 plays a crucial role in leaf responses to intracellular $\mathrm{H}_{2} \mathrm{O}_{2}$ and in ensuring appropriate gene expression through both salicylic acid and jasmonic acid signaling pathways. Plant Physiol. 153, 1144-1160. doi: 10.1104/pp.110.153767

Noctor, G., Arisi, A. C. M., Jouanin, L., and Foyer, C. H. (1998). Manipulation of glutathione and amino acid biosynthesis in the chloroplast. Plant Physiol. 118, 471-482. doi: 10.1104/pp.118.2.471

Noctor, G., and Foyer, C. H. (1998). Ascorbate and glutathione: keeping active oxygen under control. Annu. Rev. Plant Physiol. Plant Mol. Biol. 49, 249-279. doi: $\quad 10.1146 /$ annurev.arplant.49. 1.249

Noctor, G., Mhamdi, A., Queval, G., Chaouch, S., Han, Y., Neukermans, J., et al. (2012). Glutathione functions in plants: an integrated overview. Plant Cell Environ. 35, 454484. doi: 10.1111/j.1365-3040.2011. 02400.x

Noctor, G., Mhamdi, A., Queval, G. and Foyer, C. H. (2013). Regulating the redox gatekeeper: vacuolar sequestration puts glutathione disulfide in its place. Plant Physiol. doi: 10.1104/pp.113.223545 [Epub ahead of print].

Ogawa, K. (2005). Glutathioneassociated regulation of plant growth and stress responses. Antioxid. Redox Signal. 7, 973-981. doi:10.1089/ars.2005.7.973

Parisy, V., Poinssot, B., Owsianowski, L., Buchala, A., Glazebrook, J., and Mauch, F. (2007). Identification of PAD2 as a $\gamma$-glutamylcysteine synthetase highlights the importance of glutathione in disease resistance of Arabidopsis. Plant J. 49, 159-172. doi: 10.1111/j.1365-313X.2006.02938.x

Pasternak, M., Lim, B., Wirtz, M., Hell, R., Cobbett, C. S., and Meyer, A. J. (2008). Restricting glutathione biosynthesis to the cytosol is sufficient for normal plant development. Plant J. 53, 999-1012. doi: 10.1111/j.1365-313X.2007.03389.x

Pellny, T. K., Locato, V., Diaz Vivancos, P., Markovic, J., De Gara, L., Pallardó, F. V., et al. (2009). Pyridine nucleotide cycling and control of intracellular redox state in relation to poly (ADP-ribose) polymerase activity and nuclear localisation of glutathione during exponential growth of Arabidopsis cells in culture. Mol. Plant 2, 442-456. doi: $10.1093 / \mathrm{mp} / \mathrm{ssp} 008$.

Queval, G., Thominet, D., Vanacker, H., Miginiac-Maslow, M., Gakière, B., and Noctor, G. (2009). $\mathrm{H}_{2} \mathrm{O}_{2}$-activated up-regulation of glutathione in Arabidopsis involves induction of genes encoding enzymes involved in cysteine synthesis in the chloroplast. Mol. Plant 2, 344-356. doi: $10.1093 / \mathrm{mp} / \mathrm{ssp} 002$

Reichheld, J. P., Khafif, M., Riondet, C., Droux, M., Bonnard, G. and Meyer, Y. (2007). Inactivation of thioredoxin reductases reveals a complex interplay between thioredoxin and glutathione pathways in Arabidopsis development. Plant Cell 19, 1851-1865. doi: 10.1105/tpc.107. 050849

Schindelin, J., Arganda-Carreras, I., Frise, E., Kaynig, V., Longair, M., Pietzsch, T., et al. (2012). Fiji: an opensource platform for biological-image analysis. Nat. Methods 9, 676-682. doi:10.1038/nmeth.2019

Schulz, P., Neukermans, J., Van Der Kelen, K., Mühlenbock, P., Van Breusegem, F., Noctor, G., et al. (2012). Chemical PARP inhibition enhances growth of Arabidopsis and reduces anthocyanin accumulation and the activation of stress protective mechanisms. PLoS ONE 7:e37287. doi: 10.1371/journal.pone.0037287

Tzafrir, I., Pena-Muralla, R., Dickerman, A., Berg, M., Rogers, R., Hutchens, S., et al. (2004). Identification of genes required for embryo development in Arabidopsis. Plant Physiol. 135, 12061220. doi: 10.1104/pp.104.045179

Vernoux, T., Wilson, R. C., Seeley, K. A., Reichheld, J. P., Muroy, S., Brown, S., et al. (2000). The ROOT MERISTEMLESS1/CADMIUM SENSITIVE2 gene defines a glutathionedependent pathway involved in initiation and maintenance of cell division during postembryonic root development. Plant Cell 12, 97-110. doi: 10.1105/tpc. 12.1.97
Wachter, A., Wolf, S., Steiniger, H., Bogs, J., and Rausch, T. (2005). Differential targeting of GSH1 and GSH2 is achieved by multiple transcription initiation: implications for the compartmentation of glutathione biosynthesis in the Brassicaceae. Plant J. 41, 1530. doi: 10.1111/j.1365-313X.2004. 02269.x

Conflict of Interest Statement: The authors declare that the research was conducted in the absence of any commercial or financial relationships that could be construed as a potential conflict of interest.

Received: 04 July 2013; accepted: 30 September 2013; published online: 05 November 2013.

Citation: Schnaubelt D, Schulz P, Hannah MA, Yocgo RE and Foyer CH (2013) A phenomics approach to the analysis of the influence of glutathione on leaf area and abiotic stress tolerance in Arabidopsis thaliana. Front. Plant Sci. 4:416. doi: 10.3389/fpls.2013.00416

This article was submitted to Plant Physiology, a section of Frontiers in Plant Science.

Copyright (C) 2013 Schnaubelt, Schulz, Hannah, Yocgo and Foyer. This is an open-access article distributed under the terms of the Creative Commons Attribution License (CC BY). The use, distribution or reproduction in other forums is permitted, provided the original author(s) or licensor are credited and that the original publication in this journal is cited, in accordance with accepted academic practice. No use, distribution or reproduction is permitted which does not comply with these terms. 